\title{
Corela
}

Cognition, représentation, langage

HS-15 | 2014

Complémentarité des approches qualitatives et quantitatives dans l'analyse des discours

\section{Au delà de l'opposition quantitatif/qualitatif. Convergence des opérations de la recherche en analyse du discours}

Jules Duchastel et Danielle Laberge

\section{(2) OpenEdition}

\section{Journals}

Édition électronique

URL : http://journals.openedition.org/corela/3524

DOI : $10.4000 /$ corela.3524

ISSN : 1638-573X

Éditeur

Cercle linguistique du Centre et de l'Ouest - CerLICO

Référence électronique

Jules Duchastel et Danielle Laberge, « Au delà de l'opposition quantitatif/qualitatif. Convergence des opérations de la recherche en analyse du discours », Corela [En ligne], HS-15 | 2014, mis en ligne le 24 octobre 2014, consulté le 01 mai 2019. URL : http://journals.openedition.org/corela/3524 ; DOI : 10.4000/corela.3524

Ce document a été généré automatiquement le 1 mai 2019.

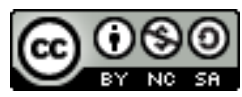

Corela - cognition, représentation, langage est mis à disposition selon les termes de la licence Creative Commons Attribution - Pas d'Utilisation Commerciale - Partage dans les Mêmes Conditions 4.0 International. 


\title{
Au delà de l'opposition quantitatif/ qualitatif. Convergence des opérations de la recherche en analyse du discours
}

\author{
Jules Duchastel et Danielle Laberge
}

\section{Introduction}

1 Il existe de grandes oppositions dans l'univers des sciences sociales et des sciences du langage: entre compréhension et explication, entre analyse structurale et analyse phénoménologique, entre différents domaines et disciplines qui prennent en compte la dimension langagière, entre traditions nationales ou continentales, entre qualitatif et quantitatif. Ces oppositions sont souvent créatrices de nouvelles avenues de pensée, mais elles deviennent stériles lorsqu'elles s'enferrent dans le renoncement à certaines dimensions de l'analyse. Nous tenterons de répondre à quelques questions : où se situe l'analyse du discours dans ce régime d'oppositions/rencontres? Que signifie la tension qualitatif/quantitatif et peut-on la dépasser dans la mixité des méthodes? En quoi l'interprétation ne peut se distinguer de l'objectivation des opérations de la recherche?

Dans un premier temps, nous montrerons en quoi l'analyse du discours constitue un point de confluence entre disciplines, traditions et approches. Nous aborderons, par la suite, l'opposition entre méthodes qualitatives et quantitatives et la solution que propose l'approche des méthodes mixtes. Cela nous conduira à revoir l'opposition entre expliquer et comprendre afin d'établir l'existence, dans toute science, d'un " arc herméneutique " qui ne dissocie pas l'interprétation de l'explication. Nous montrerons, à travers la description des transformations du texte dans le processus d'analyse du discours, les nécessaires réduction et restauration de la complexité de l'objet propre à toute démarche quantitative ou qualitative. Nous illustrerons en terminant la compatibilité des méthodes en montrant que les concepts de causalité et de mesure s'appliquent aussi bien dans le cadre de l'une ou de l'autre approche. 


\section{Oppositions et convergences en Analyse du discours}

3 L'analyse du discours se situe à la confluence de diverses disciplines, traditions, approches. Elle est née d'une double nécessité de dépasser, dans les sciences humaines, la simple analyse de contenu et, dans les sciences du langage, l'approche structurale et abstraite de la langue. L'analyse du discours introduit l'impératif de considérer la langue dans son actualisation sociale et de penser le contenu en tant qu'il se réalise dans le fonctionnement et la forme linguistiques. On peut décrire cette rencontre comme fusion de deux grandes traditions: les sciences humaines ou sociales en tant que sciences herméneutiques, lesquelles s'appuient sur le sens donné aux pratiques et institutions sociales et les sciences du langage qui s'attardent sur la description du fonctionnement des différents aspects de la langue en usage. Il faut souligner, dans le contexte de cette confluence, l'apparition d'un troisième axe, celui des mathématiques et de l'informatique, qui donnera lieu au développement d'une tradition d'analyse du discours assistée par ordinateur. Si on peut difficilement parler de l'analyse du discours comme d'une discipline, c'est bien en raison de ce foisonnement d'influences qui produit autant de pratiques d'analyse qu'il y a de disciplines et de types d'entrecroisements entre elles.

Schéma I: Confluences en Analyse du Discours

4 On peut quand même présenter un tableau d'ensemble de ces convergences qui tient compte des oppositions déjà mentionnées. Le schéma 1 représente l'ensemble des oppositions et des convergences des diverses traditions d'analyse telles qu'elles sont nées autour des années soixante ${ }^{1}$. Le schéma présente en son centre l'analyse du discours comme point de confluence de l'ensemble de ces traditions. Cela n'en fait pas une discipline, mais un champ de pratique de la recherche qui partage un certain nombre de conceptions en provenance de plusieurs disciplines. Cette confluence est également marquée par les échanges nombreux entre traditions nationales. Le schéma peut être lu comme un ensemble d'oppositions, de haut en bas, de gauche à droite et selon les diagonales. La première grande opposition, de haut en bas, distingue les approches qualitatives et quantitatives. Dans des sens très différents, il est possible de considérer les approches occupant le haut du schéma comme appartenant de manière générale à une logique qualitative, celle des lettres (Pires, 1982), alors que le bas du schéma représente les approches quantitatives favorisant les chiffres. La deuxième grande opposition du schéma distingue, de gauche à droite, les traditions française et anglo-saxonne ${ }^{2}$, en même temps qu'elle met en évidence la prépondérance respective des sciences du langage d'un côté et des sciences sociales de l'autre.

5 On obtient au point de départ un carré où chacun des termes s'oppose aux autres, aussi bien horizontalement, verticalement que diagonalement. Si on se situe au haut du schéma, dans la perspective dite qualitative, ce sont l'École française d'analyse du discours et la tradition anglo-saxonne d'analyse qualitative qui forment la première opposition. Ce qui les distingue avant tout est leur appartenance respective à des traditions disciplinaires différentes. L'analyse du discours à la française trouve son ancrage dans la linguistique distributionnelle, fonctionnelle et l'analyse de l'énonciation qui ont pour point commun d'élargir l'étude de la langue à son usage situé. Elle doit beaucoup à la tradition structuraliste qui veut appréhender les phénomènes symboliques 
dans leur dimension systémique. Elle développe peu à peu une réflexion sur le discours en tant qu'objet théorique et objet empirique (Foucault, 1969) et évoluera progressivement vers une linguistique du texte (Rastier, 2001; Adam, 1999).

6 De l'autre côté, l'analyse qualitative se développe dans le giron de l'interactionnisme symbolique et de la phénoménologie, mais également dans celui de la philosophie du langage et de la pragmatique. Ces traditions ont un intérêt commun pour l'action intentionnelle qui se manifeste à travers des faits de langage. Alors que la tradition française met l'accent sur la dimension linguistique du discours situé, la tradition américaine s'intéresse au langage comme vecteur de la construction sociale de la réalité. Ce qui manifeste particulièrement la différence entre les deux traditions est le type de discours qui est pris en compte. La tradition française s'intéresse au point de départ aux discours institutionnels, politiques ou littéraires. La tradition américaine favorise le discours ordinaire, les interlocutions localisées, la conversation.

7 On peut de la même manière, opposer dans l'axe inférieur du schéma, soit celui de l'approche quantitative, la tradition française d'analyse lexicométrique et la tradition américaine d'analyse de contenu. Ces deux approches partagent un même intérêt pour la quantification et la mesure des phénomènes langagiers, mais elles se distinguent par leur origine même. Alors qu'en France, on note un intérêt pour la statistique appliquée aux champs littéraire et politique, en Amérique, c'est l'étude de la communication et de la propagande qui a donné naissance à une tradition d'analyse de contenu. Au point de départ, dans les deux cas, c'est la croyance en la puissance d'explication des chiffres qui constitue la base de l'approche. Les modèles mathématiques et statistiques différeront en raison même de la méthode. D'un côté, on applique des méthodes de plus en plus complexes aux mots dans leur existence "naturelle », c'est-à-dire non catégorisés, de l'autre, on favorise le décompte relativement simple des unités du langage en tant qu'elles sont codées. Mais dans les deux cas, ce qui importe est d'accéder au sens du message à travers les nombres.

8 Si l'on observe le schéma selon l'axe vertical, il est possible de distinguer, du côté gauche en France, l'analyse du discours et l'analyse lexicométrique. On passe progressivement d'un dialogue de sourds entre les deux écoles, dans les années soixante et soixante-dix, à une reconnaissance réciproque au cours des récentes années, à mesure que les systèmes d'analyse du discours assistée par ordinateur commencent à imposer leur propre légitimité. Tout se passe comme si les exigences de formalisation des procédures informatiques rendaient moins "rébarbatifs » des calculs statistiques aux yeux de ceux d'abord intéressés par la description des fonctionnements langagiers. Du côté droit, dans la tradition américaine, on observe une même opposition entre méthode qualitative et méthode quantitative. Dans les deux cas, on s'intéresse avant tout au sens des énoncés, mais la tradition qualitative privilégie la lecture interprétative qui procède de la catégorisation des énoncés, alors que l'analyse de contenu s'intéresse, du moins à ses débuts, au décompte essentiellement quantitatif des unités du discours. Cette opposition s'est également atténuée au cours des ans et on ne trouve plus guère de chercheurs parfaitement orthodoxes. À preuve, l'introduction progressive dans les logiciels d'analyse qualitative de fonctionnalités de décompte.

9 Enfin, dans les axes diagonaux du schéma, on peut opposer, deux à deux, les traditions. Il est clair que l'opposition entre lexicométrie et analyse qualitative découle de la même logique que celle qui oppose les deux approches, quantitative et qualitative, d'analyse de contenu aux États-Unis. Mais, cette opposition n'est pas très thématisée dans les écrits. 
Celle qui l'est davantage est celle qui met face à face analyse du discours et analyse de contenu. Elle a pris forme dans l'acte fondateur de l'analyse du discours en France. On se souvient que l'origine même de la tradition française d'analyse du discours s'est appuyée sur la critique de la tradition d'analyse de contenu (Haroche \& coll., 1971). Cette critique a beaucoup insisté sur l'ignorance du substrat linguistique du discours dans la tradition américaine, bien que certains auteurs, comme Osgood (1959), en aient justifié toute l'importance.

10 L'analyse de discours, comme pratique de recherche, a toujours eu un caractère syncrétique, chacune des traditions puisant à plusieurs sources disciplinaires et méthodologiques. Il en découle que les oppositions décrites ci-devant furent à l'origine d'une confluence entre les diverses perspectives. Cela est vrai du rapprochement, en France, entre les traditions d'analyse du discours et de lexicométrie, maintenant désignée sous le label de textométrie ou de logométrie ${ }^{3}$. Cela est aussi vrai de la conjugaison des méthodes d'analyse de contenu, qualitatives et quantitatives aux États-Unis. De même, les traditions française et américaine d'analyse du discours se sont fortement rapprochées dans les dernières décennies. Ce qui les distinguait au point de départ, la nature des discours analysés (politique dans un cas et de la vie quotidienne dans l'autre) et l'origine disciplinaire (linguistique pour l'une et pragmatique pour l'autre), a progressivement convergét. Il est intéressant de noter que les auteurs de référence des traditions anglosaxonnes d'analyse critique du discours (Fairclough) ou d'analyse des représentations sociales (Stuart Hall) sont les mêmes que celles de l'École française d'origine, soit LéviStrauss, Barthes, Althusser, Derrida et Foucault. Tout aussi intéressant est de constater que l'analyse de la connaissance ordinaire et de la conversation a traversé l'Atlantique dans l'autre sens. Il n'est pas question de conclure à l'unité du champ en analyse du discours, mais de constater que la pratique même d'analyse combine de plus en plus les disciplines, les approches et les méthodologies.

\section{Mixité des méthodes}

11 La confluence des courants théoriques et méthodologiques qui cerne les contours du champ de pratique de l'analyse du discours implique la mixité des méthodes. Le concept même de mixité des méthodes s'inscrit plus largement dans le projet de dépasser l'opposition entre approche qualitative et approche quantitative, de combiner en quelque sorte les deux méthodes. Si le quantitatif est relativement facile à définir, il n'en est pas de même du qualitatif. Par exemple, dans l'opposition entre la partie supérieure gauche et la partie supérieure droite de notre schéma, il y a déjà deux façons d'envisager l'approche qualitative. Une première façon consiste à dire que les méthodes d'analyse du discours s'emploient à un travail de description des formes et du fonctionnement linguistiques du discours, en somme qu'elles prennent en compte des «qualités » du discours. La seconde renvoie plus proprement à l'approche qualitative comme telle. Mais, avant d'en venir à la définition des paradigmes quantitatif et qualitatif, il est bon de souligner le contraste entre les deux projets d'analyse. Alors que l'on peut définir le quantitatif de manière assez simple, comme utilisation «d'outils d'analyse mathématiques et statistiques, en vue de décrire, d'expliquer et de prédire des phénomènes par le biais de concepts opérationnalisés sous forme de variables mesurables $»^{5}$, le qualitatif renvoie à un très grand nombre de pratiques de recherche, tels que les énumèrent Denzin et Lincoln (1994) : étude de cas, ethnographie, observation 
participante, phénoménologie, ethnométhodologie, théorie ancrée, méthode biographique, recherche-action, recherche clinique.

C'est donc du côté des paradigmes de recherche que l'opposition entre quantitatif et qualitatif prend sens. On peut définir trois plans qui caractérisent chaque paradigme: épistémologique, analytique et opérationnel. Ces configurations peuvent se décliner de différentes manières selon les postures ontologiques des différents chercheurs, mais elles indiquent de manière générale des positions communes devant la tâche qu'ils se donnent. Nous n'approfondirons pas ici la question de la posture ontologique quant à l'existence de la réalité et de la vérité qui se situe en amont des positions épistémologiques. Ces postures, positiviste, néo ou post positiviste, critique ou constructiviste, accordent à la réalité une existence plus ou moins autonome. Il en est de même du régime de vérité dont le degré de relativité s'accroît sur l'axe allant du positivisme au constructivisme ${ }^{6}$. Ces postures influencent nécessairement la position empruntée par chacun aux divers plans paradigmatiques. Voyons ce qu'il en est des deux paradigmes ${ }^{7}$.

Reprenons chacun des plans épistémologique, analytique et opérationnel qui caractérisent les deux paradigmes qualitatif et quantitatif. Ils forment une suite d'oppositions qui mériteraient d'être abondamment discutées. Mais, l'objectif ici est de donner un aperçu des principaux débats qui existent entre les deux points de vue. Au plan épistémologique, on peut distinguer trois questions. La première question concerne le point de vue du sujet de connaissance : alors que l'approche quantitative adopterait un point de vue positiviste ou néo-positiviste en préconisant la mise à distance du sujet de connaissance et de l'objet étudié ainsi que l'objectivité des procédures, l'approche qualitative favoriserait l'empathie et la subjectivité. La seconde question concerne la possibilité de généralisation: le quantitatif viserait la formulation de propositions générales et universelles alors que le qualitatif insisterait sur la singularité et le contexte. La troisième question porte sur la valeur de vérité : le quantitatif viserait la validité des procédures et la neutralité de l'observateur. Le qualitatif substituerait à l'idée de validité, celle de «transférabilité » et de crédibilité et à celle de neutralité, l'engagement axiologique.

Au plan de la démarche analytique, le quantitatif procèderait à la réduction de la complexité plutôt qu'à son rétablissement. Il favoriserait une approche déductive, au moins dans la phase confirmatoire, alors que le qualitatif mettrait de l'avant une démarche inductive ou abductive. Par ailleurs, le quantitatif encouragerait des analyses en largeur (thin) plutôt qu'en profondeur (thick) qui caractériseraient le qualitatif. Enfin, au plan des opérations, le quantitatif travaillerait sur des variables alors que le qualitatif s'intéresserait aux actions intentionnelles. L'objectif du quantitatif serait la mesure alors que le qualitatif s'intéresserait aux processus. En conséquence, le quantitatif viserait les tests statistiques confirmatoires et le qualitatif les procédures exploratoires. En somme, le but du quantitatif serait l'explication causale et celui du qualitatif la compréhension du sens.

Le recours aux méthodes mixtes s'explique par un affaiblissement de cette opposition paradigmatique entre quantitatif et qualitatif et l'adoption d'une attitude plus pratique. Conscients de la nature variable des données et de leur simple disponibilité, les chercheurs en sont venus à recourir à des matériaux ou à des approches analytiques qu'on aurait eu tendance à opposer antérieurement. Ces changements se fondent la plupart du temps sur des arguments essentiellement pragmatiques. Cela donne des résultats! Un examen des pratiques dans ce domaine montre qu'il y a pour l'essentiel trois 
perspectives pour procéder à ces combinaisons. Une première perspective peut être qualifiée de fonctionnelle. Elle consiste simplement à juxtaposer l'usage des divers types de méthodes selon les besoins de la recherche et la nature des données. Le choix pour le chercheur revient à établir la séquence des emplois de méthodes qualitatives et quantitatives et leur importance respective (QUAN>qual ; QUAL>quan; QUAN=QUAL) dans le cadre de la démarche. La deuxième perspective est plutôt substantive. Il s'agit de construire des hybridations des méthodes en raison de l'objet même d'étude. Par exemple, l'analyse du discours et l'analyse de contenu s'appliquent à des phénomènes comprenant a priori des aspects à la fois qualitatifs et quantitatifs. La troisième perspective est de nature démonstrative et son usage des méthodes mixtes se fonde sur l'idée de la triangulation des méthodes. La triangulation vise à accroître la confiance dans les résultats de la recherche. L'usage du terme triangulation est cependant métaphorique (Kelle, 2001) et ne garantit pas formellement une validité plus grande, sinon sous forme de convergence ou de confirmation des conclusions. En somme, l'usage des méthodes mixtes ne fait qu'introduire le constat de la non-mutuelle exclusion des types de méthodes. Il nous semble pourtant insuffisant d'un point de vue méthodologique de réduire la question des méthodes mixtes à leur seule efficacité sans tenter de saisir les conséquences des oppositions épistémologiques, analytiques et opérationnelles caractérisant les deux paradigmes qualitatif et quantitatif sur ces nouvelles formes de démarches empiriques.

\section{Expliquer et comprendre}

Deux conclusions découlent de ce qui précède. D’une part, nous avons établi que la pratique d'analyse du discours s'inscrit dans la confluence de plusieurs disciplines qui, elles-mêmes, adoptent des approches plus ou moins quantitatives ou qualitatives, structurales ou phénoménologiques, linguistiques ou sociologiques. Alors que chaque tradition d'analyse s'est établie sur la base d'oppositions épistémologiques, théoriques ou méthodologiques avec les autres traditions, on constate aujourd'hui une certaine convergence des méthodes et une atténuation des fractures. D'autre part, l'opposition fondamentale entre méthodes qualitatives et quantitatives semble se dissoudre dans l'approche pragmatique des méthodes mixtes. Ce pragmatisme fait l'économie d'une réflexion ontologico-épistémologique sur les fondements de cette approche mixte. La question devient de savoir si les deux paradigmes qui semblent s'exclure mutuellement peuvent être réconciliés.

17 Afin d'élucider cette question, il est utile de revenir au point de départ de la séparation des sciences naturelles et des sciences de l'esprit telle que l'a établie Dilthey à la fin du 19 ème siècle. Cette séparation se caractérisait par une opposition ferme entre explication et compréhension. Selon cette conception, les sciences naturelles étaient tout entières tournées vers l'identification de relations causales entre phénomènes, alors que les sciences humaines cherchaient à débusquer le sens de l'expérience vécue historiquement située. C'est bien cette conception qui se trouve à la base de l'opposition paradigmatique entre méthodes quantitative et qualitative. Nous reprendrons l'hypothèse de Ricœur (1986: 162) selon laquelle « expliquer et comprendre ne constitueraient pas les pôles d'un rapport d'exclusion, mais les moments relatifs d'un processus complexe qu'on peut appeler interprétation. » En fait, Ricœur définit un arc herméneutique, allant de l'explication à la compréhension, c'est-à-dire que l'interprétation se déploie dans un 
ensemble de procédures objectives d'observation, de description et d'analyse qui aboutissent à la compréhension de l'objet. L'herméneutique n'est pas interprétation immédiate de la réalité observée, comme ce pourrait être le cas de la connaissance ordinaire. Dans la connaissance scientifique, l'interprétation est nécessairement appuyée sur la médiation de procédures pouvant être dites explicatives.

Cette hypothèse nous permet de récuser deux conceptions courantes de l'interprétation. La première s'inscrit au sein même du paradigme qualitatif où l'interprétation relève souvent $\mathrm{du}$ commentaire herméneutique. Elle est le fait de certains manuels qui définissent l'analyse qualitative "comme une représentation et une transposition conscientes, délibérées et rigoureuses du système "soi-monde-autrui" de manière à en faire une nouvelle exploration dans l'optique particulière des sciences humaines et sociales, lesquelles s'efforcent de faire surgir le sens en rendant compréhensible » (Paillé \& Mucchielli, 2008). Le travail vise à faire ressortir les significations en contexte, à interpréter le discours. En fait, c'est l'unique fonction référentielle du discours qui domine ici. Mais justement, le propre de l'analyse du discours est de mettre en évidence les divers aspects linguistiques et paralinguistiques du discours dont la description est nécessaire à sa compréhension globale. L'interprétation ne peut donc se tenir seule et nécessite un travail de description et d'explication.

La seconde conception de l'interprétation que nous récusons est celle qui se restreint aux seuls résultats. Que ce soit dans un cadre quantitatif ou qualitatif, on limite le travail de l'interprétation comme le sens donné aux résultats produits par les opérations de la recherche. On entretient alors l'illusion d'une pure objectivité de ces opérations qui serait maintenue jusqu'au moment d'attribuer un sens aux résultats qu'elles produisent. C'est négliger l'importance des actes interprétatifs qui accompagnent chacune des étapes du processus de recherche. La projection d'un cadre théorique, l'identification des dimensions de l'analyse, les choix des valeurs de propriétés prêtées à l'objet sont autant d'actes interprétatifs logés au sein même des procédures d'objectivation.

Qu'est-ce donc alors que l'interprétation? Dans l'acception large, on a tendance à confondre ce concept avec la compréhension, soit l'appropriation pour soi du sens d'une action, d'une intention ou d'une pensée. Le chercheur serait alors appelé à développer ses aptitudes empathiques, susceptibles de lui donner accès à la conscience des sujets observés. Il est vrai qu'au terme de chaque recherche, l'auteur en arrive à une interprétation globale du phénomène observé qui est en quelque sorte détachée de l'ensemble des procédures d'observation, de description et d'analyse des données. Cette interprétation globale se rapproche de ce qu'on peut concevoir comme une appropriation pour soi, une compréhension du phénomène (Duchastel \& Laberge, 1999a). Mais, dans le cadre d'une activité scientifique, l'interprétation doit être vue comme un processus de confrontation du chercheur avec la matérialité discursive (Conein et coll., 1981) ou, autrement dit, la matière langagière (Paveau, 2012). On trouve chez plusieurs auteurs, cette intuition forte selon laquelle l'accès au sens ne peut esquiver la matérialité discursive. Outre Pêcheux et Paveau, on trouve chez Molino (1989) l'idée d'une trace du discours qui est seule accessible à l'analyse. De même, Ricœur (1986: 141) parle de « l'occultation du monde circonstanciel par le quasi-monde des textes » comme condition de la lecture et de l'interprétation. En somme, l'herméneutique en tant qu'art de l'interprétation est fondée sur un ensemble de procédures de description et d'analyse des unités matérielles du discours. 
21 Cela fut l'intuition à la base du projet d'analyse du discours qui proposait, dès son origine, de dépasser l'analyse de contenu en tenant compte de la dimension linguistique du discours. Le discours n'en fut pas pour autant réduit aux dimensions proprement linguistiques - lexicale, ou sémantique. L'hypothèse consistait à rechercher les différentes marques ou traces des diverses fonctions du discours telles que les a développées par exemple Jakobson, au cœur même de la matérialité langagière. Il en est ainsi de l'analyse de l'énonciation qui cherche l'inscription du locuteur et des allocutaires dans le fil du discours. Il en est encore de même des analyses de l'argumentation qui identifient des marqueurs caractérisant le processus argumentaire. Si l'on suit Gee (2011) dans sa définition de l'analyse du discours, on peut affirmer que le discours fait l'objet à la fois d'une analyse de l'information qu'il véhicule, de l'action qu'il suscite et de l'identité qu'il formule. Chacune de ces dimensions n'est repérable qu'à travers des formes langagières qui les rendent intelligibles. L'interprétation doit donc s'appliquer à définir certaines classes d'unités d'observation ${ }^{8}$ et proposer une description de leurs propriétés. L'analyse est, en cela, un processus interprétant à l'aide de ressources d'objectivation.

Devant de telles propositions, une conception restrictive de l'interprétation ne peut plus être soutenue. L'interprétation ne peut être restreinte au terme du processus de recherche, au moment où le chercheur se retrouve devant des résultats. Il faut plutôt inverser le raisonnement. Si l'interprétation repose sur des procédures objectives, ces dernières ne peuvent à leur tour faire l'économie de l'interprétation. Cela revient à dire qu'objectivation et interprétation vont de pair et ne s'opposent pas comme semble le suggérer la querelle des paradigmes. Plutôt que de concevoir deux approches générales définies par leur finalité, expliquer ou comprendre, il faut les intégrer au sein d'un même processus. Aucune science ne peut faire l'économie d'une précompréhension de l'objet. Il $\mathrm{y}$ a toujours un cadre de connaissance, prenant une forme plus ou moins théorique, qui oriente la saisie du réel. Et, en finalité, c'est une compréhension augmentée qui est recherchée. L'explication revêt toutes sortes de formes. Elle peut en effet être conçue, comme nous le dit Kaplan (1964), comme explication sémantique, c'est-à-dire comme une interprétation. On explique le sens d'un énoncé. L'explication est le plus souvent pensée comme relation de détermination entre deux phénomènes. À chaque fois, l'objectif est de mieux comprendre. Les diverses procédures d'observation, de description et d'analyse des objets visent à accroître la compréhension en distinguant la mise à distance de l'objet et sa mise en relation avec les cadres cognitifs du sujet de connaissance.

Il faut cependant souligner l'asymétrie des processus d'objectivation et d'interprétation. Alors que les procédures d'objectivation peuvent être maîtrisées, l'acte d'interprétation, même s'il est bien isolé, demeure difficilement définissable. On pense ici aux capacités cognitives variables du chercheur aux plans sémantique, émotionnel ou culturel qui ont pour résultat une certaine indétermination de l'interprétation. Il est cependant plus facile de maîtriser le processus interprétatif dans les divers actes de description et d'analyse que dans celui de la compréhension d'ensemble d'un phénomène. Nous distinguons ainsi les interprétations locales qui peuvent être réfléchies, sinon maîtrisées, à toutes les étapes du processus de la recherche et les interprétations globales qui redonnent sens à la complexité au détriment d'une maîtrise assurée des opérations cognitives à l'œuvre ( Duchastel \& Laberge, 1999a). 


\section{Le problème de la complexité}

24

l'approche réductrice qui le caractériserait au détriment de la complexité. Le paradigme compréhensif s'appuierait, au contraire, sur l'idée que la complexité des phénomènes doit être saisie par l'analyste. Ce paradigme manifeste une forte résistance au découpage des objets qui risquerait d'en dissiper le sens. C'est donc une approche empathique et compréhensive qui est prônée. Mais est-il possible d'appréhender un objet sans le découper et le décrire? Les méthodes qualitatives n'échappent pas à cette exigence puisqu'elles procèdent, elles-mêmes, à l'identification d'unités plus ou moins larges (segments textuels, phrases, paragraphes) auxquelles elles apposent des catégories descriptives. Les tenants du paradigme qualitatif insistent pourtant sur la nécessité de ne jamais sacrifier la totalité aux parties.

La question est peut-être mal posée. Il faut plutôt distinguer la réduction méthodique de la complexité et la simplification. Certes, la distinction entre analyse en profondeur (thick ) et en largeur (thin) demeure pertinente et on conçoit bien que la première embrasse davantage de complexité. Mais, dans tous les cas, il est inévitable de procéder à une certaine réduction des dimensions du phénomène à l'étude. Il n'est pas possible de saisir un objet dans sa globalité, sinon de manière intuitive. Il faut nécessairement suspendre temporairement l'examen de certaines de ses composantes pour n'en retenir que quelques-unes. Ricœur (1986) nous explique que l'analyse du discours ne peut se faire qu'à travers la médiation du texte. Il s'agit d'un acte méthodique qui, grâce à l'occultation de la complexité des conditions du discours, permet l'identification d'une matérialité textuelle, l'observation de ses propriétés et des relations entre elles. Cet acte de nature mixte, explicative et interprétative, ne conduira à une compréhension plus globale qu'au terme du processus de la recherche.

26 Nous concevons le processus de compréhension comme une spirale formée de cercles superposés comportant chacun un point d'origine fondé sur une précompréhension de l'objet et un point d'aboutissement défini comme compréhension enrichie du même objet. Entre les deux points, un ensemble d'opérations de construction, de description et d'analyse de l'objet s'appuient sur une dynamique mettant en jeu l'explication et l'interprétation. Il s'agit d'un travail d'identification des dimensions et des unités de l'objet, de description de ces unités en fonction des dimensions conceptuelles et de mise en relation de ces unités. L'ensemble de ces opérations ne peut se faire que sur une matérialité définie. Cette matérialité est celle du texte et le texte n'est jamais qu'une trace du discours en situation. On peut déjà dire que le texte est ainsi une certaine réduction de l'objet discours. Mais, il n'est pas possible de procéder à l'analyse sans recourir au support textuel, sinon dans le processus naturel de compréhension ordinaire.

Schéma 2 : Les transformations des formes du texte

Les transformations des formes du texte au fil de l'analyse nous permettront de comprendre comment s'opère un double processus de réduction et de restauration de la complexité. Le schéma $2^{9}$ indique les diverses formes que prendra le texte à chacune des opérations méthodiques propres à l'analyse du discours. La première forme est celle du texte Discours. Il représente la matière brute à partir de laquelle nous allons effectuer les diverses opérations de la recherche. Le texte discours est, au point de départ, un objet 
complexe inscrit dans un ensemble de conditions de production relevant du contexte sociohistorique, cognitif, culturel ou linguistique et dépendant des conditions d'énonciation propres à la situation de communication. La première transformation vise à établir le texte Manuscrit. À l'origine, il peut s'agir d'un discours oral ou d'un discours écrit, déjà sous la forme d'un texte. Dans le cas du discours écrit, il faut sélectionner et authentifier une version du discours qui formera un texte "hors du monde» pour reprendre l'expression de Ricœur. Dans le cas du discours oral, il faudra procéder à sa transcription. Le discours oral comporte un ensemble de caractéristiques prosodiques ou contextuelles qui peuvent être notées de manière plus ou moins élaborée à partir de conventions ${ }^{10}$. Le texte Manuscrit est donc un objet à la fois différent et moins complexe que le discours d'origine dans le sens où les conditions de sa production ou de son énonciation ne sont plus repérables qu'en son sein même.

La transformation suivante consiste à produire la version du texte Édition. Quelle que soit la situation des textes manuscrits, transcrits de l'oral, en format papier ou sur support informatisé, un travail de normalisation doit être effectué afin de rendre comparables les divers éléments d'un corpus. Les diverses informations concernant les conditions de production et d'énonciation (locuteur, support, lieu, temps, etc.) doivent accompagner chaque document d'un corpus. Nous obtenons ainsi un nouveau texte Édition qui pourra faire l'objet des opérations subséquentes de description, d'exploration et d'analyse. En résumé, le texte Manuscrit est une dérivation du discours originel dont la version manuscrite a dû être établie par des opérations d'authentification ou de transcription et le texte Édition est, à son tour, le résultat d'un travail de normalisation et d'indexation selon un système de règles et de catégories descriptives. C'est sur la base de ce texte Édition que le travail d'observation et de description s'accomplira.

En quoi consiste l'analyse de la matérialité discursive ainsi établie? Elle comporte deux tâches initiales: la définition des unités d'observation, ce que l'on veut observer; la description de ces unités en fonction d'un ou de plusieurs systèmes de catégories, comment on observe. Les unités d'observation peuvent être représentées comme un ensemble d'éléments emboîtés, allant du corpus, aux sous-corpus, à l'ensemble des textes qui les constituent, aux diverses parties des textes jusqu'aux méso et micro unités des textes. Chaque niveau d'emboîtement des unités du corpus pourra être caractérisé. Le corpus lui-même et ses sous-ensembles sont indexés par un système de métadonnées. Chaque texte peut être marqué selon les constituantes du texte (section, paragraphe, échanges verbaux, etc.). Enfin, des unités d'observation du discours (segments textuels, tours de parole, phrases, mots) reçoivent des catégories en fonction du type de description visé (par exemple, morphosyntaxique, sémantique, pragmatique, énonciative, argumentative). Se dégage ainsi un système de description des données se déployant à trois niveaux: celui du corpus décrit par les métadonnées, celui des parties du texte décrit par des variables structurelles, celui des unités du discours décrites par une variété de propriétés associées à la problématique de recherche. On peut affirmer alors que le texte édité se transforme en texte Représentation, au sens où le texte est désormais enrichi de descriptions et, d'une certaine façon, où la complexité est partiellement restaurée. Il ne s'agit plus cependant du texte originel et on ne peut prétendre qu'il corresponde entièrement au contexte de son énonciation.

Toutes les descriptions du texte pourront être triées et compilées. Elles pourront faire ou non l'objet de décomptes, de croisements, de comparaisons en fonction des divers segments établis sur la base des métadonnées ou des variables structurelles. Chacune des 
opérations d'exploration des données décrites donnera lieu à la production d'autant de nouveaux textes prenant la forme de commentaires ou de résultats chiffrés. Chacun de ces Sous-textes ne sera qu'une image lointaine du texte originel. C'est le cumul de ces images qui permettra l'exploration approfondie du discours initial et conduira à l'interprétation des données, produisant une nouvelle transformation sous la forme du texte Interprétation. L'interprétation des résultats variera en généralité selon que l'on interprète les données empiriques produites par les différentes explorations ou que l'on tente de donner un sens global aux données, cette fois prises dans leur ensemble. Le texte Interprétation mobilisera alors beaucoup plus que le dispositif méthodologique mis en place, pour restaurer la complexité du discours à l'aide des connaissances sociohistoriques et théoriques du chercheur. Le texte Interprétation prendra la forme d'un article ou d'une monographie dont l'objectif sera désormais la compréhension du phénomène étudié.

31 Cette représentation plus ou moins métaphorique d'une succession des états du texte vise à montrer que le discours ne peut s'appréhender que sous la forme de la matérialité textuelle et que celle-ci doit nécessairement faire l'objet d'opérations méthodiques. De ce point de vue, il ne nous semble pas pertinent de distinguer les méthodes quantitatives et qualitatives. Sur le plan épistémologique, il ne nous semble pas opportun d'opposer complexité et réduction. Il s'agit en effet d'une autre manière d'opposer compréhension et explication. La démarche méthodologique implique nécessairement une réduction de l'objet autorisant une certaine objectivation des données. Comme nous l'avons vu plus haut, ce processus associe en tout temps les opérations d'explication et d'interprétation. Celles-ci conduisent en fin de compte à la formulation d'hypothèses interprétatives qui permettent l'appropriation pour soi de l'objet, c'est-à-dire sa compréhension.

\section{Causalité et mesure en analyse du discours}

Nous avons tenté, jusqu'à présent, de montrer en quoi l'analyse du discours est, comme son nom l'indique, une pratique d'analyse qui porte sur une matérialité discursive et qui met en œuvre des opérations méthodiques, à la fois explicative et interprétative. Nous avons récusé les oppositions trop faciles entre paradigmes qualitatif et quantitatif tout en reconnaissant l'existence de pratiques distinctives s'intéressant aux aspects quantitatif ou qualitatif des phénomènes. L'opposition paradigmatique entre les approches qualitative et quantitative insiste sur deux critères distinctifs. Comme nous l'avons souligné, le quantitatif privilégierait la mesure et l'explication causale; quant au qualitatif, il se fonderait plutôt sur le processus et la compréhension. Pour se convaincre de la compatibilité des deux approches, il est utile d'examiner la présence du raisonnement causal dans la pratique d'analyse du discours et la pertinence de la mesure comme opération de réduction et de restauration de la complexité dans l'analyse. Nous tenterons d'illustrer successivement que la causalité et la mesure ont leur place dans l'approche qualitative.

En ce qui concerne la causalité, nous reprendrons la thèse proposée par Tacq (2010), sur la présence du raisonnement causal dans la recherche aussi bien quantitative que qualitative. Ce dernier donne un aperçu des différentes théories de la causalité dans les sciences sociales pour en retenir l'idée d'une logique expérimentale présente dans les deux démarches. Il part de la prémisse qu'en science, la relation causale est rarement appréhendée de manière directe, mais qu'elle est plutôt envisagée dans une démarche 
indirecte, une sorte de processus d'encerclement. Ainsi, les sciences recourent le plus souvent à la démarche probabiliste des statistiques ou encore à l'examen des conditions nécessaires et suffisantes pouvant expliquer un phénomène, sans pour autant pouvoir établir un lien direct de causalité entre phénomènes. Pour appuyer sa conception de la logique expérimentale, Tacq s'appuie sur le modèle INUS ${ }^{11}$ de Mackie (1974) qui détermine la nature du raisonnement, fondé sur l'ensemble des conditions qui rendent possible l'occurrence d'un événement.

Selon le modèle INUS, un événement peut être le produit de conditions nécessaires, mais insuffisantes de manière générale, tout en étant suffisantes bien que non nécessaires dans les circonstances. Reprenons l'exemple donné par Tacq. Des experts peuvent déclarer qu'un incendie est le résultat d'un court-circuit. La cause ne peut être déclarée nécessaire parce que d'autres facteurs pourraient causer l'incendie. Elle ne peut être déclarée suffisante dans la mesure où d'autres conditions peuvent contribuer à la propagation du feu. Tout ce qu'on peut dire, c'est qu'il y a un ensemble de conditions positives ou négatives qui, associées au court circuit, sont suffisantes, sans pour autant être non nécessaires pour déclencher l'incendie. Il s'agit d'un raisonnement contrefactuel qui interroge la possibilité de l'occurrence d'un événement en l'absence du facteur identifié comme étant causal. On est alors dans la perspective d'un champ causal et non dans celle d'une causalité logique. Selon l'auteur, ce type de raisonnement est largement pratiqué dans la recherche de nature expérimentale. C'est la même logique qui serait mise en œuvre dans la recherche qualitative.

Afin d'appuyer sa thèse, Tacq répond aux principaux arguments visant à distinguer les démarches qualitative et quantitative. Le premier concerne les niveaux de mesure correspondant aux échelles nominales, ordinales, par intervalles et métriques. Les deux premiers niveaux, nominal et ordinal, caractéristiques de l'approche qualitative, seraient, en raison de la nature des opérations mathématiques permises, exclus de la logique causale impliquée par les modèles quantitatifs. S'il est vrai que les opérations mathématiques varient en fonction de la nature des variables, il n'en résulte pas que la logique causale en est de facto exclue. Le deuxième argument s'appuie sur la différence de la taille des échantillons entre les approches qualitative et quantitative. À l'extrême, des études qualitatives vont travailler sur des cas uniques, ce qui rendrait l'analyse causale problématique. L'auteur répond à cet argument, en soulignant qu'il existe peu de critères objectifs pour déterminer la taille minimale d'un échantillon et que même l'analyse d'un cas unique peut faire sens, pourvu qu'il soit mis en relation avec d'autres études de cas unique. L'analyse du complexe des conditions nécessaires et suffisantes est toujours possible par l'examen contrefactuel de ces conditions. Le troisième argument concerne la possibilité d'effectuer des tests statistiques. Évidemment, la puissance des tests statistiques variera grandement en fonction de la taille des échantillons. Néanmoins, il existe une panoplie de tests qui ont été produits pour valider les résultats de petits échantillons et la comparaison des données avec d'autres données, obtenues dans d'autres études, constitue en soi une sorte de test, même s'ils ne sont pas de nature statistique. Le dernier argument porte sur la différence entre analyse en largeur (thin) et en profondeur (thick). Encore une fois, nul doute que l'analyse en profondeur multiplie les dimensions observées de l'objet, alors que l'analyse en largeur multiplie les individus observés sur un nombre limité de dimensions. Cela ne devrait pourtant pas changer le raisonnement, d'autant plus que rien n'empêche de combiner des stratégies qualitatives et quantitatives à diverses étapes de la recherche. 
36 L'auteur conclut que si l'on retient l'approche contrefactuelle et conditionnelle du modèle INUS (Mackie, 1974) et la méthode des différences à la base de l'approche expérimentale telle que formulée par Stuart Mill, il n'y a pas de différence de principe entre les méthodes quantitatives et qualitatives du point de vue du raisonnement causal.

37 Nous terminerons en montrant que l'utilisation de la mesure n'est pas non plus incompatible avec l'approche qualitative ${ }^{12}$. Si l'on s'en rapporte au paradigme qualitatif, la mesure contribuerait à la dénaturation de l'objet observé et conduirait à produire des analyses fallacieuses et inutiles, en raison même de la réduction qu'elle induirait de la complexité. Nous tenterons de montrer, au contraire, que l'application de la mesure constitue l'une des opérations de la recherche permettant une certaine abstraction des dimensions de l'objet étudié et, éventuellement, la reconstitution d'un autre ordre de complexité. Nous retiendrons la définition que propose Kaplan (1964 : 207) : «La mesure est l'assignation de nombres à des objets, des évènements ou des situations à partir d'un système de règles définissant des propriétés pouvant être quantifiées. » Les propriétés de l'objet ainsi que leur mesurabilité n'existent pas indépendamment d'une théorie. La représentation qualitative ou quantitative d'un objet dépend du choix d'un système de représentation symbolique. Pour reprendre la formulation de Kaplan, « les quantités sont celles des qualités et la mesure d'une qualité n'a d'autre valeur que celle qui est exprimée dans la mesure. » En somme, la mesure peut s'appliquer à divers degrés de construction de l'objet. En premier lieu, elle peut porter sur tout objet ayant une existence matérielle indépendante, quelle qu'en soit la nature, la taille ou la complexité, par exemple les individus, les objets du monde, les textes, les énoncés, les évènements. Deuxièmement, elle peut s'appliquer à des segments ou propriétés de ces objets qui ne sont pas directement accessibles à l'observation, mais qui découlent d'un travail de construction. Troisièmement, la mesure peut même porter sur des objets immatériels dont l'existence relève du travail de l'esprit, que cette production soit une production sociale (le succès, la richesse, la popularité...) ou qu'elle soit le produit d'une tradition ou d'une communauté disciplinaire (l'anomie, le relativisme social, la créativité...).

Pour reprendre notre propos antérieur, la mesure peut, en effet, se présenter comme une opération de réduction de l'information. Dans les actes qui conduisent à la mesure, on renonce à certains attributs de l'objet pour ne retenir que ceux jugés pertinents à la démarche. Il s'agit d'un travail d'abstraction fondé sur la renonciation à la diversité des manifestations concrètes, matérielles ou imaginées, de l'objet. Ce travail d'abstraction est présent dans les deux démarches qualitative ou quantitative. Elle se traduit dans les opérations de description et de catégorisation des unités retenues. La catégorisation suppose une double réduction de l'objet par l'identification d'un aspect de l'objet et par l'attribution d'une valeur abstraite capable de le représenter. Le fait de compter les valeurs des unités et de leurs propriétés succède au travail préalable de réduction et d'abstraction des dimensions de l'objet. En contrepartie, la mesure peut également contribuer à restituer la complexité. Elle peut en effet constituer une stratégie heuristique puissante visant à reconstruire des représentations complexes des aspects ou attributs postulés sur le plan théorique. Par exemple, la construction d'indices visant à représenter un concept par l'addition et la pondération d'indicateurs donne lieu à l'émergence d'une forme de complexité non apparente au point de départ. De même manière, les analyses statistiques multidimensionnelles donnent à voir des observations de l'objet qui ne sont pas a priori possibles. 
L'analyse du discours représente un bon exemple pour l'application de la mesure dans le cadre d'une approche mixte. Si l'on revient aux opérations de description et d'analyse des données, on constate que l'application de la mesure contribue à la fois au travail d'abstraction des dimensions de l'objet et au rétablissement de la complexité. Tout effort d'analyse de discours procède au découpage du discours en unités discrètes (des mots, des syntagmes sémantiquement significatifs, des segments textuels plus larges...) et détermine un système de catégorisation (sémantique, sociologique, argumentatif, énonciatif...). Libre au chercheur de déterminer s'il tiendra compte uniquement des unités, quel qu'en soit le niveau de découpage, ou s'il s'intéressera aux propriétés de ces unités. Les décomptes effectués de ces objets donneront un aperçu partiel de l'objet dans sa totalité. Il serait possible d'apprendre, par exemple, quelle est la part des substantifs appartenant à une classe sémantique, quelles prémisses dominent dans une structure argumentaire, quelle est l'importance relative des marqueurs énonciatifs dans un discours politique, quelle est la fréquence des tours de paroles dans une conversation, etc. C'est ainsi que l'on peut parler d'une lecture réductrice qui se manifeste à la fois par une certaine sélection des aspects du texte et sa représentation dans un système de mesure. Mais, il est aussi possible de parler d'une complexification de la représentation du texte par la multiplication des observations et le cumul des éléments mesurés. Le cumul des observations et des mesures peut conduire à la construction d'indices ou à la multiplication des dimensions de l'analyse. La mesure ne constitue alors qu'une opération disponible dans la panoplie des moyens permettant l'analyse. Elle n'est nullement par nature incompatible avec la démarche qualitative.

\section{Conclusion}

Nous avons montré que l'analyse du discours n'est pas une discipline, mais un champ de pratique qui se situe à la confluence d'un ensemble de traditions disciplinaires et nationales. Ce lieu privilégié explique la richesse des héritages disciplinaires, théoriques et méthodologiques. L'objet même de l'analyse du discours la prédispose à se loger à la rencontre des démarches d'analyse au sein même de ce que l'on peut appeler les méthodes mixtes. Nous avons montré que les oppositions paradigmatiques entre les approches qualitative et quantitative, bien que fortement défendues dans le corpus des écrits scientifiques, s'épuisaient dans le recours pragmatique à une sorte de mixité de leur emploi. Nous avons voulu aller au-delà de cette attitude pragmatique, pour défendre la thèse qu'il existe bel et bien un fond commun à la démarche méthodologique, quel que soit son rattachement paradigmatique. Nous avons ainsi montré qu'on ne peut interpréter sans expliquer et que les opérations d'identification des unités de la recherche et de description et d'analyse de ces unités combinent en tout temps objectivation et interprétation. Nous avons de plus indiqué que la connaissance scientifique ne peut procéder sans appliquer des procédures de réduction, mais que la combinaison de ces procédures peut conduire à une restauration de la complexité de l'objet. Nous avons terminé en montrant que la logique de la causalité et de la mesure, qui dans la querelle des écoles semble opposée aux méthodes qualitatives, s'applique aussi bien à l'une ou l'autre démarche qualitative et quantitative. 


\section{BIBLIOGRAPHIE}

Adam J.-M., 1999. Linguistique textuelle. Des genres de discours aux textes, Paris : Nathan.

Althusser L., 1970. Idéologie et appareils idéologiques d'état. La Pensée, 151, Juin.

Barthes R., 1957. Mythologies, Paris : Seuil.

Conein B., Courtine J.-J., Gadet F. \& Pêcheux M., 1981. Matérialités discursives. Lille: Presses Universitaires de Lille.

Denzin N. K. \& Lincoln Y. S. (Eds), 1994. Handbook Of Qualitative Research. London : Sage.

Derrida J., 1967. L'écriture et la différence, Paris: Seuil.

Duchastel J. \& Laberge D., 1999a. Des Interprétations Locales Aux Interprétations Globales : Combler Le Hiatus. In N. Ramognino \& G. Houle (Dir.) Sociologie et normativité scientifique. Toulouse: Presses Universitaires Du Mirail, 51-72.

Duchastel J. \& Laberge D., 1999b. La recherche comme espace de médiation interdisciplinaire. Sociologie et Sociétés, Xxxi : 1, 63-76.

Duchastel J. \& Laberge D., 2011. La mesure comme représentation de l'objet. Analyse et interprétation. In Sociologies, avril, Http://Sociologies.Revues.Org.

Fairclough N., 2007. Discourse And Social Change. Cambridge : Polity, 225-240.

Foucault, M., 1969. L'archéologie du savoir. Paris : Gallimard.

Jefferson G., 2004. Glossary Of Transcript Symbols With An Introduction. In G. Lerner (Ed.), Conversation Analysis: Studies From The First Generation. Amsterdam, Netherlands : John Benjamins Pub., 13-31.

Gee J. P., 2011. An Introduction To Discourse Analysis, Theory And Method. $3^{\text {ème }}$ Édition, New York : Routledge.

Guba E. G. \& Lincoln Y. S., 1994. Competing Paradigms In Qualitative Research. In N. K. Denzin \& Y. S. Lincoln (Eds), Handbook Of Qualitative Research. London : Sage.

Haroche C. L., Henri P. \& Pêcheux M., 1971. La Sémantique et la coupure saussurienne : langue, langage, discours. Langages, 24.

Harris Z., 1952. Discourse Analysis. Language, 28 : 1, 1-30.

Jakobson R., 1963. Essais de linguistique générale. Paris: Minuit.

Kaplan A., 1964. The Conduct Of Inquiry. Methodology For Behavioral Science. New York: Chandler Publishing.

Kelle U., 2001. Sociological Explanations Between Micro And Macro And The Integration of Qualitative And Quantitative Methods. Forum: Qualitative Social Research, 2:1.

Mackie J., 1974. The Cement Of The Universe. A Study Of Causation. Oxford : Oxford University Press.

Mayaffre D., 2007. Analyses logométriques et rhétoriques des discours. In S. Olivési (Dir.), Introduction à la recherche en sic. Grenoble : Presses Universitaires de Grenoble, 153-180. 
Meunier J.-G., 1990. Le traitement et l'analyse informatique des textes. Gestion De L'information Textuelle. Ico, 2 : Iii, 9-18.

Foucault M., 1970. L'archéologie du savoir. Paris : PUF.

Hall S. (Ed.), 2009. Representation, Cultural Representations And Signifying Practices. London : Sage.

Molino J., 1989. Interpréter. In C. Reichler (Dir.), L'interprétation des textes. Paris, Editions de Minuit, 9-52.

Osgood C. E., 1959. The Representational Model And Relevant Research Methods. In I. S. Pool (Ed.), Trends In Content Analysis Urbana : Univ. Of Illinois Press.

Paillé P. \& Mucchielli A., 2008. L'analyse qualitative en sciences humaines et sociales. Paris : Armand Colin.

Paveau M.-A., 2012. L'alternative quantitatif/qualitatif à l'épreuve des univers discursifs numériques. Colloque international et interdisciplinaire Complémentarité des approches qualitatives et quantitatives dans l'analyse des discours? Amiens, France.

Pêcheux M., 1975. Les vérités de La Palice, linguistique, sémantique, philosophie. Paris: Maspero. Pires A., 1982. La méthode qualitative en Amérique du nord : un débat manqué (1918-1960). Sociologie et société, $14: 1,16-29$.

Rastier F., 2001. Arts et sciences du texte. Paris : PUF.

Ricœur P., 1986. Du texte à l'action. Paris: Seuil.

Tacq J., 2011. Causality In Qualitative And Quantitative Research. Quality And Quantity, 45 : 2, 263-291

Zienkowski J., 2012. Overcoming The Post-Structuralist Methodological Deficit, Metapragmatic Markers And Interpretative Logic In A Critique Of The Bologna Process. Pragmatics, $22: 3$, 501-534.

\section{ANNEXES}

Références dans Schéma 1

Althusser L., 1970. Idéologie et appareils idéologiques d'état. La Pensée, 151, Juin.

Austin J. L., 1962. How To Do Things With Words, Oxford Univ. Press, New York, 1962 ( Quand Dire, C'est Faire, Seuil, Paris, 1970).

Barthes R., 1957. Mythologies. Paris : Seuil.

Benveniste E., 1966. Problèmes de linguistique générale. Vol.1, Paris : Gallimard.

Benzecri J-P., 1973. L'analyse des données: l'analyse des correspondances. Paris : Dunod.

Berelson B., 1952. Content Analysis In Communication Research. New York: Hafner Publ.

Berger P. \& Luckman T., 1966. The Social Construction Of Reality: A Treatise In The Sociology of Knowledge. Garden City, N.Y. : Anchor Books.

Dubois J., 1969. Énoncé et énonciation. Langages, 4 : 13 , 100-110.

Foucault M., 1969. L'archéologie du savoir. Paris: Gallimard. 
Garfinkel H.,1967. Studies In Ethnomethodology. Englewood Cliffs, N. J. : Prentice-Hall

(Trad. franç. : Recherches en ethnométhodologie, Paris : PUF, 2007).

Guiraud P., 19600. Problèmes et méthodes de la statistique linguistique. Paris : PUF.

Harris Z., 1952. Discourse Analysis. Language, 28 : 1, 1-30.

Hjelmslev L., 1947. Structural Analysis Of Language. Studia Linguistica, 1 : 1-3, 69-78.

Holsti O. R., 1969. Content Analysis For The Social Sciences And Humanities. Addison Wesley.

Lacan J., 1966. Écrits. 2 Volumes. Paris : Seuil.

Laswell H. D., Merner D. \& Pool I., 1952. The Comparative Study Of Symbols. Stanford

University Press.

Lévi-Strauss C., 1949. Les structures élémentaires de la parenté. Paris : PUF.

Mead G. H., 1934. Mind, Self, And Society,. Ed. Charles W. Morris. Chicago : U. Of Chicago Press.

Muller C., 1968. Initiation à la statistique linguistique. Paris : Larousse.

Pêcheux M., 1969. Analyse automatique du discours. Paris: Dunod.

Sacks H., 1972. An Initial Investigation Of The Usability Of Conversational Data For Doing Sociology. In D. Sudnow (Ed.) Studies In Social Interaction. New York Free Press, 31-74.

Schütz A., 1967. The Phenomenology Of The Social World. Evanston : Northwestern University Press.

Searle J., 1970. Speech Acts: An Essay In The Philosophy Of Language. Cambridge : Cambridge Universtiy Press.

Stone P. S., Dunphy D. C., Smith, M. S. \& Ogilvie D. M., 1966. The General Inquirer, A Computer Approach To Content Analysis In The Behavioural Sciences. Cambridge, Mass: M.I.T. Press.

\section{NOTES}

1. Nous avons retenu les noms et dates de publication des œuvres séminales des auteurs ayant marqué de manière significative chacune des traditions. Cette liste est indicative et ne saurait être exhaustive.

2. Notons que l'opposition entre école française et tradition anglo-saxonne n'est pas parfaitement hermétique. Par exemple, l'École française d'analyse du discours s'inspire au départ des travaux de Z. Harris en linguistique distributionnelle.

3. Dans les récentes années en France, on a tendance à substituer au terme lexicométrie, ceux de textométrie (Rastier, 2001) et de logométrie (Mayaffre, 2007), afin de signifier le caractère étendu des unités du langage qui peuvent être prises en charge par les logiciels.

4. Par exemple, Zienkowski, (2012) montre la nécessité de palier le déficit méthodologique de la tradition post-structuraliste de l'analyse du discours (tradition d'Essex) par le recours aux outils de la pragmatique.

5. Wikipédia, http://fr.wikipedia.org/wiki/M\%C3\%A9thodes_quantitatives

6. Voir la table 6.2. "Paradigm positions on Selected Practical Issues", dans Guba \& Lincoln, Y. S. (1994) et le tableau 1. "Trois positions ontologiques dans les sciences sociales contemporaines » dans Duchastel \& Laberge, 1999 b. 
7. Notons que ces paradigmes sont définis à la manière d'idéaltypes. Il n'est pas dit que chacune des assertions qu'ils comportent font l'objet d'un accord dans la communauté des chercheurs qui adoptent l'une ou l'autre approche.

8. Nous revenons à la question de la définition des unités à la section 5 .

9. Le schéma 2 est dérivé d'un schéma présenté par Meunier (1990).

10. Ex. Gale Jefferson (2004).

11. Insufficient but Necessary part of a set, which is Unnecessary but Sufficient for the Result.

12. Les propos qui suivent s'inspirent largement de Duchastel \& Laberge (2011).

\section{RÉSUMÉS}

Après avoir montré que l'analyse du discours n'est pas une discipline, mais un champ de pratique qui se situe à la confluence d'un ensemble de traditions disciplinaires et nationales, nous proposons de renoncer à l'opposition tranchée entre approches qualitative et quantitative au profit de la mixité des méthodes. Non seulement la pratique de la recherche montre qu'il ne saurait y avoir une mutuelle exclusion des types de méthodes, mais ces dernières se réfèrent à un schème commun de la connaissance qui mobilise des opérations partagées de la recherche. Nous montrerons qu'expliquer et comprendre ne sont pas des processus contradictoires et que l'interprétation scientifique ne peut tenir indépendamment de toute opération explicative. Toute démarche scientifique, qualitative ou quantitative, repose sur un fond commun mobilisant des opérations d'identification des unités de la recherche, de leur description et de leur analyse. S'il est vrai que les paradigmes analytiques divergent quant à leurs présupposés épistémologiques et méthodologiques, ils se trouvent confrontés au même problème de la réduction et de la restauration de la complexité. À titre d'exemple limite, nous illustrons en quoi les questions de la causalité et de la mesure se posent dans tout raisonnement scientifique.

Having shown that discourse analysis is not a discipline but a field of practice that lies at the confluence of a set of national and disciplinary traditions, we propose to abandon the sharp opposition between qualitative and quantitative approaches to the benefit of mixed methods. Not only does research show that there can be no mutual exclusion between types of methods, but all methods refer to a common pattern of knowledge that involves research operations that are shared. We show that explaining and understanding are not contradictory processes and that scientific interpretation can not stand independently of any explanatory operation. Any scientific process, qualitative or quantitative, is based on a common ground mobilizing research operations for the identification of units, their description and their analysis. While the analytical paradigms differ on their epistemological and methodological assumptions, they are facing the same problem of reducing and restoring complexity. We conclude in showing how the issues of causality and measurement arise in all scientific reasoning, wathever their nature, qualitative or quantitative. 
INDEX

Mots-clés : analyse du discours, quantitatif/qualitatif, méthodes mixtes, explication, interprétation, opérations de recherche, complexité, causalité, mesure.

Keywords : discourse analysis, quantitative / qualitative, mixed methods, explanation, operations research, complexity, causality, measurement.

\section{AUTEURS}

JULES DUCHASTEL

Centre ATO UQAM - Canada

DANIELLE LABERGE

Centre ATO UQAM - Canada 